Philosophie ANTIQUE
Philosophie antique

Problèmes, Renaissances, Usages

13 | 2013

Analogie et connaissance

\title{
Federico M. PETRUCCI, Teone di Smirne, Expositio rerum mathematicarum ad legendum Platonem utilium
}

Joëlle Delattre

\section{OpenEdition}

\section{Journals}

Édition électronique

URL : https://journals.openedition.org/philosant/916

DOI : 10.4000/philosant.916

ISSN : 2648-2789

\section{Éditeur}

Éditions Vrin

\section{Édition imprimée}

Date de publication : 1 novembre 2013

Pagination : 269-273

ISBN : 978-2-7574-0591-8

ISSN : $1634-4561$

Référence électronique

Joëlle Delattre, «Federico M. PETRUCCI, Teone di Smirne, Expositio rerum mathematicarum ad legendum Platonem utilium », Philosophie antique [En ligne], 13 | 2013, mis en ligne le 01 novembre 2018, consulté le 03 décembre 2022. URL : http://journals.openedition.org/philosant/916 ; DOI : https://doi.org/ 10.4000/philosant.916

\section{cc) (i) (2)}

Creative Commons - Attribution - Pas d'Utilisation Commerciale - Pas de Modification 4.0 International - CC BY-NC-ND 4.0

https://creativecommons.org/licenses/by-nc-nd/4.0/ 
Federico M. PETRUCCI, Teone di Smirne, Expositio rerum mathematicarum ad legendum Platonem utilium. Introduzione, Traduzione, Commento, Sankt Augustin, Academia Verlag, 2012 (Studies in Ancient Philosophy, 11), 609 p. ISBN 9783896655509.

D'un versant à l'autre des Alpes, les règles universitaires et éditoriales diffèrent beaucoup, et c'est une aubaine pour les chercheurs et les érudits. Voici Théon de Smyrne, élégamment propulsé sur le devant de la scène par un jeune chercheur de 27 ans, dans une traduction italienne actualisée, commentée au fil du texte à la mode antique, avec une volonté non dissimulée d'exhaustivité dans les références, les parallèles, les contrastes et les variantes, les interprétations divergentes. Les notes de bas de page foisonnantes de l'introduction et du commentaire ouvrent chacune de nouveaux dossiers, documentés avec la plus grande érudition, aussi bien sur la tradition arabe et l'époque médiévale que sur les commentateurs de l'Antiquité tardive ou de l'époque moderne. Des tableaux synoptiques (p. $46-49$ et $52-53$ de l'introduction) pour les thèmes des recherches musicales et astronomiques, puis (p. 542-552, à la fin du commentaire) pour les recherches arithmétiques et (p. 553555) d'autres sujets astronomiques, sont un modèle du genre et prouvent l'aisance extrême avec laquelle l'auteur sait aussi faire se correspondre les textes des auteurs anciens.

Le livre comporte 609 pages en caractères serrés et de petite taille, avec des marges réduites. La bibliographie y occupe 18 pages dans un ordre strictement alphabétique, sans distinguer les éditions de textes anciens des écrits généraux, ni des articles spécialisés sur chacune des disciplines concernées par cette ample recherche. Elle est suivie d'un index des sources antiques (20 pages), puis d'un index analytique (13 pages) qui mêle des termes généraux, des termes techniques et des noms d'auteurs anciens, où les références à la traduction sont distingućes de celles du commentaire, enfin d'un bref index des termes grecs (2 pages), proposant un choix réduit de concepts, sans que soient toujours indiqués les sens adoptés dans la traduction.

La présentation introductive fort dense ( 53 pages) est immédiatement suivie des notes philologiques sur le texte et la traduction ( 38 pages), argumentées avec le même scrupule érudit, pour justifier ou contredire les corrections apportées ou non aux manuscrits par les éditeurs et traducteurs précédents (Boulliau, De Gelder, Martin, Hiller, Dupuis, Smyly, Tannery, Barker et même Delattre). Le texte grec (96 pages), en effet, est ensuite donné en bloc (avec les corrections adoptées et les figures de l'édition Teubner), simplement complété dans la marge de droite par les numéros de page de Hiller en gras et les numéros de ligne de cinq en cinq; puis la traduction italienne ( 86 pages) est donnée aussi d'un seul bloc, enchaînant sans même un passage de ligne l'Introduction, l'Arithmétique, la Musique et l'Astronomie de Théon de Smyrne, ponctuées des seuls repères entre parenthèses de la pagination de Hiller en caractères gras, et des numéros de lignes de dix en dix.

Chaque rubrique du commentaire (229 pages) est ensuite introduite simplement par les numéros de pages et de lignes de l'édition de référence, si bien que le lecteur est contraint de se reporter au texte et à la traduction correspondante, au fur et à mesure de sa lecture : marque-page ou post it recommandés! Quatre sous- 
titres néanmoins y respectent cette fois la tétralogie de Théon, et certaines références soulignées en début de paragraphe semblent renvoyer (mais pas toujours) aux larges divisions proposées dans la présentation : I (p. 19), II (p. 21-22), III (p. 26) et IV (p. 31), sans que cela ait été précisé.

Trois Appendices complètent encore le commentaire. Le dernier (5 pages) porte sur le style mathématique traditionnel de Théon et s'interroge sur certaines de ses impropriétés; le deuxième ( 6 pages) étudie dans le détail la citation du mythe d'Er pour montrer que les nombreuses variantes ne sont pas seulement du ressort de l'Art of Misquotation, mais ont une raison « idéologique » par rapport à l'objectif de Platonem ex Platone $\sigma \alpha \varphi \eta \nu i \zeta \varepsilon \iota \nu$ (p. 536). Quant au premier (16 pages), il étudie avec précision, en se référant en parallèle au Commentaire de Calcidius (sans toutefois renvoyer jamais aux travaux de Béatrice Bakhouche ${ }^{1}$ ), la manière dont Théon de Smyrne utilise le Commentaire sur le Timée d'Adraste d'Aphrodise. L'auteur y utilise la même méthode « au fil du texte » que dans son commentaire et finit par renvoyer à l'un de ses articles, «plus complet » sur la question (p. 530, n. 34). La complexité des arguments et le caractère touffu de leur présentation explique sans doute que, dans sa traduction, l'auteur ait renoncé à faire figurer en italiques les citations d'Adraste comme celles des nombreux autres auteurs cités (ou à utiliser des guillemets comme dans la traduction française ${ }^{2}$ ). Cela entraîne du coup des ambiguités dans le commentaire, qui attribue parfois à Théon les arguments que ce dernier prête explicitement à Adraste, jusqu'à le taxer ensuite d'incohérence, dès lors qu'il va prendre justement ses distances par rapport à la citation qu'il a donnée. Or, un des enjeux principaux de l'écrit astronomique de Théon est bien, et l'auteur le formule avec pertinence, de faire saisir au lecteur ce qui distingue le modèle astronomique platonicien qu'il promeut (le mouvement planétaire retardé ou inversé, mouvement vrai, correspond à la révolution de l'Autre par rapport à celle du Même), du modèle péripatéticien d'Adraste qu'il utilise néanmoins abondamment (le mouvement planétaire observé est un mouvement apparent, une $\phi \alpha v \tau \alpha \sigma i \alpha^{3}$ ou un фasvóusvov dont il faut rendre compte).

La tonalité scrupuleusement traditionnelle de cette somme remarquable, probablement induite par l'obligation universitaire de se soumettre aux critères d'évaluation des maitres, en cherchant même à devancer leurs attentes, et de satisfaire aux canons de la recherche académique sur les auteurs antiques sans prendre le risque d'en braver l'immobilisme, procurera toutefois au lecteur intéressé et curieux d'aujourd'hui quelques sujets d'étonnement, voire de déception.

Par exemple, le choix des termes latins (utilisés dans la traduction de la fin du $\mathrm{XIX}^{\star}$ s. par J. Dupuis) pour désigner les rapports « d'un plus une part » (par quoi

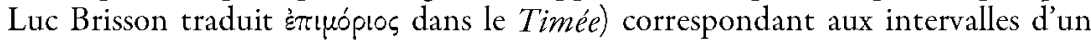

1. Cf. B. Bakhouche, Calcidius, Commentaire au Timée de Platon (2 vol.), Paris, Librairie philosophique J. Vrin, 2012.

2. Cf. J. Delattre Biencourt, Théon de Smyme, Lire Platon. Le recours au savoir scientifique: Arithmétique, Musique, Astronomie, Toulouse, Anacharsis, 2010.

3. Cf. D. et J. Delattre, «L La phantasia des planètes dans la Moyenne Antiquité », dans Incontri Triestini di Filologia classica, IV, 2004-2005, Atti del Convegno Internazionale: Phantasia. Il pensiero per immagini degli antiqui e dei moderni, p. 315-334. 
ton $(1+1 / 8)$ et d'une quinte $(1+1 / 2)$, conserve au texte un caractère vieillot: pourquoi ne pas avoir gardé simplement les termes grecs comme pour l'intervalle de quarte, rendu par « épitrite $»(1+1 / 3)$ ou pour le rapport « d'un plus plusieurs parts », transcrit en «épimère »? De la même manière, le choix de conserver le titre latin de l'édition d'E. Hiller, sans le traduire sur la page de garde, par ailleurs superbement illustrée par un fragment de frise du Parthénon, et de nommer l'ouvrage de Théon, dans les pages qui suivent, « l'Expositio » sans plus, après s'être contenté de donner en grec dans la note 2 les deux versions du titre transmises par les manuscrits $A$ et $B$, est finalement assez décevant - s'il est vrai que la traduction par « Exposition » ou par « Présentation » résulte d'une lecture possible de $\chi p \eta-$ $\sigma i \mu \alpha^{4}$ au lieu de $\chi p \eta \sigma i \mu \omega \nu$, dans $B$, éventualité dont il n'est même pas question.

Mais l'objet principal de «courroux platonique», comme dirait Amyot traduisant Plutarque, tient au rejet d'une interprétation, qualifiée de «in chiave mecanica $\gg($ p. 43), des théories harmonique et astronomique mises au point par Théon comme « complètement inopportune », sans autre forme de procès ni de discussion. Pas de sphères armillaires platoniciennes construites pour soutenir les exercices de recherche des sens de rotation et de révolution les plus adéquats pour rendre compte des phénomènes observés et des points de stabilisation de tout le système ; pas de canon musical pythagoricien calibré pour donner prise aux exercices de déplacement d'un curseur par-dessus ou par-dessous, afin de remplir les intervalles entre les notes fixes au moyen des notes mobiles ajustées; pas même de simple table numérique qui permettrait de lire directement les nombres consonants et leurs multiples ainsi que les intervalles qui leur correspondent ? L'auteur réussit à commenter la nature «purement arithmogéométrique » de l'arithmétique de Théon (p. 332 sqq.) sans se référer du tout aux travaux de M. Caveing, et signale même (p.336 n. 139) un risque de « surinterprétation de l'ouvrage » qui prêterait au médio-platonicien une connaissance approfondie des implications (et des utilisations techniques) de la matière traitée, pour lui préférer une «lecture légère $\gg$ !

Nous n'insisterons donc pas sur la traduction, légère et subreptice, de l'expression récurrente dans l'écrit astronomique de Théon, $x \alpha \tau \dot{\alpha}$ ov $\mu \beta \varepsilon \beta \eta \kappa o ́ \varsigma$, traduction encore une fois calquée sur l'expression latine per accidens transmise par l'astronomie médiévale, et qui ne tient pas compte de la Note détaillée que Th. Martin a consacrée à ce sujet. Passer complètement sous silence cette dernière difficulté ne peut être «accidentel »! Cela provoque toutefois un certain flottement dans le commentaire: ainsi, p. 483, l'auteur convient que «le modèle de l'excentrique s'ensuivrait du modèle selon l'épicycle per accidente (autrement dit, serait nécessairement impliqué par lui) », avec l'explicitation entre parenthèses, tandis que, p. 496, il n'est plus question que de la démonstration (considérée comme partielle) de « la coïncidence accidentelle du modèle de l'excentrique avec celui de l'épicycle $\gg$.

Enfin, le choix très judicieux de proposer en un tableau comparatif les principaux objets de recherche ou ל๊

4. Cf. J. D. Macadam, Theo Smymaeus On Arithmetics, Thesis for the Degree of Master of Arts, University of British Columbia, 1969. 
ordre d'apparition dans le Timée de Platon et dans les passages correspondants de «l'Expositio », avec en vis-à-vis leur traitement « technique » et leur traitement «exégétique », met en évidence deux autres biais de l'étude entreprise. Tout d'abord, l'ordre dans lequel Théon aborde les différents thèmes est loin de suivre celui du Timée, et les écarts donnent précisément la mesure de la réécriture et de la recomposition du propos platonicien par le commentateur médio-platonicien, ce que le commentaire proposé «au fil du texte» ne permet pas de bien appréhender. Ensuite, l'utilisation systématique de la batterie des Y̧ๆ de fait comme une remise en ordre plus traditionnel du propos théonien, au risque d'en occulter l'originalité. Certes, il est indéniable que Théon de Smyrne se situe explicitement dans une tradition technique largement exploitée (néopythagoricienne et ératosthénienne pour l'arithmétique et l'harmonie, hipparquienne et eudoxéenne pour l'astronomie); mais sa pratique de l'exégèse médio-platonicienne, en s'inscrivant dans l'espace intermédiaire entre l'écrit du péripatéticien Adraste qu'il cite et critique longuement avant de se référer de manière bien plus expéditive, mais aussi critique, à l'écrit du platonicien Dercyllide, ne se pose-t-elle pas justement comme une revendication explicite d'originalité ?

Selon l'auteur (p.60, n. 178), une double caractéristique de l'exégèse médioplatonicienne tiendrait à la fois dans « l'extension de la reformulation exégétique aux conséquences philosophiques des écrits platoniciens» et dans «l'élargissement possible (sans crainte de falsification) attribuant à Platon le corpus entier des connaissances techniques » qui permettra en réalité de « réaffirmer paradoxalement $\gg$ son autorité (p. 61). Autant l'extension aux conséquences se perçoit bien, par exemple, dans le traitement qui est fait des citations de Platon dans la première partic de la tétralogie théonienne, aussi bien pour la métaphore des teinturiers que pour le parallèle entre l'apprentissage des disciplines scientifiques et les stades de l'initiation aux Mystères, autant l'élargissement falsificateur est loin d'être aussi évident. L'exemple le plus flagrant d'erreur astronomique et d'amalgame commis par Théon de Smyrne correspond sans doute à l'effort pour distinguer « avec plus d'exactitude $\gg$ (Hiller 172.22) trois mesures «très proches » du retour périodique du Soleil au même point, que «la plupart des mathématiciens astronomes considèrent comme égales à 365 jours et quart $\gg$. Les commentateurs ont beaucoup glosé sur ce passage pour pointer l'incompétence scientifique de Théon ou le «bas niveau » de ses sources. L'auteur examine tout cela avec soin (p. 486); mais pourquoi se limite-t-il ici aux seuls arguments de type technique, sans se demander à quel «transfert exégétique » cela peut correspondre ? Les trois temps de retour du soleil au même point «à la même saison» correspondent peut-être à des phénomènes astronomiques connus et mesurables aujourd'hui, mais les valeurs de 365 jours et demi et 365 jours $1 / 8$ semblent être plutôt de simples écarts de mesure d'un seul et même phénomène, lequel a provoqué la réforme, encore récente pour Théon, du calendrier julien. De fait, ils sont surtout l'occasion pour le médioplatonicien d'introduire les durées, purement «théoriques » cette fois, de deux, quatre et huit ans $\left(2,2^{2}\right.$ et $\left.2^{3}\right)$ associables aux trois mouvements en longitude, latitude et profondeur, afin que mouvements et durées correspondent «plus exactement $\gg$ à la progression de la première tétractys platonicienne (1-2-4-8), 
branche droite du lambda de sept nombres que Platon utilise pour structurer l'harmonie fondamentale de l'âme du monde.

Sans aller plus avant dans les détails du commentaire, c'est sans hésitation que nous reconnaissons la qualité soignée de cette publication très rapidement menée à terme et l'utilité indéniable de ce travail très richement documenté, fourni par un jeune chercheur tout à fait érudit, pour tous les connaisseurs - mathématiciens, musiciens, astronomes, philologues ou philosophes - de Théon de Smyrne.

Joëlle DeLATTRE

D. E. Machuca (éd.), New Essays on Ancient Pyrrhonism, Leyde/Boston, Brill (Philosophia Antiqua 126), 2011, XI + 207 p. ISBN 9789004207769.

Le volume offre huit articles sur le scepticisme pyrrhonien. Les essais, de la plume de M. Bonazzi, T. O'Keefe, J. Warren, F. Grgić, H. Thorsrud, S. Marchand, D. E. Machuca et O. Bueno, sont tous en anglais. Précédés d'une préface, d'une notice sur les auteurs et d'une brève introduction, ils sont suivis d'index des noms propres, des passages et des sujets. Chaque contribution est munie d'une bibliographic.

Les deux premiers articles sont consacrés à la section des Esquisses Pyrrboniennes (PH I, 209-241) où les différences entre pyrrhonisme et philosophies voisines sont discutées. En 221-225, Sextus soutient que la position de Platon n'est pas assimilable au pyrrhonisme - la remarque sextienne étant introduite, dans les

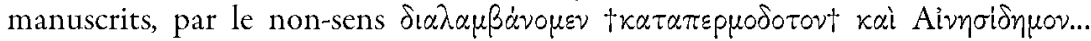
ótı... (222). Sextus soutient-il ceci en accord avec ses prédécesseurs pyrrhoniens Énésidème en particulier? La contribution de Bonazzi s'efforce de répondre à cette vexata quaestio par l'affirmative en quatre mouvements. Primo, il est judicieux d'accepter l'émendation au texte de Spinelli («Sextus Empiricus, the Neighbouring Philosophies and the Sceptical Tradition $\gg$ dans J. Sihvola [éd.], Ancient

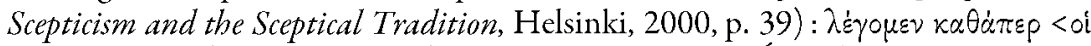

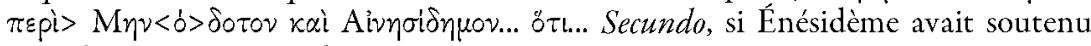
que Platon était un pyrrhonien, Sextus aurait contesté cette interprétation - ce qui n'est pas le cas. (Un mouvement à revoir ? Cet argumentum e silentio, discutable en soi, est affaibli par la prémisse douteuse selon laquelle les remarques de Sextus, en 222, ne veulent pas démontrer que Platon possédait des $\delta \dot{\delta} \gamma \mu \alpha \tau \alpha$.) Tertio, l'argument de Sextus contre l'interprétation sceptique de Platon en 222 trouve un parallèle dans ceux d'Énésidème contre le scepticisme académique (Photius, Bibliothéque, cod. 212, 170a31-38): Sextus et Énésidème semblent mobiliser des arguments semblables contre la même cible - l'Académic. Quarto, deux témoignages souvent utilisés pour montrer que certains pyrrhoniens considéraient Platon comme un collègue sceptique, l'un tiré d'un commentaire anonyme au Théétète de Platon, l'autre d'une introduction néoplatonicienne à sa philosophie, ne parlent pas des pyrrhoniens, mais des académiciens.

En $P H$ I, 215, Sextus rapporte la thèse selon laquelle cyrénaiques et sceptiques disent que nous apprenons seulement nos affects. Si la position cyrénaïque est bien attestée, sa contrepartie sceptique est problématique : Sextus ne la commente pas et en PH I, 200-201, la nie implicitement. Quelle est donc la différence entre les 